\title{
Fis binding to the recombinational enhancer of the Hin DNA inversion system
}

\author{
Michael F. Bruist, ${ }^{3}$ Anna C. Glasgow, ${ }^{1}$ Reid C. Johnson, ${ }^{1,2}$ and Melvin I. Simon ${ }^{1}$ \\ ${ }^{1}$ Division of Biology, California Institute of Technology, Pasadena, California 91125 USA; ${ }^{2}$ Department of Biological \\ Chemistry, UCLA School of Medicine, Los Angeles, California 90074 USA
}

\begin{abstract}
The recombinational enhancer of the Hin inversion system in Salmonella stimulates recombination in vitro 150-fold in the presence of the Escherichia coli host factor Fis. To gain an understanding of the roles of the enhancer and Fis in stimulating the Hin-mediated inversion reaction, we have used nuclease and chemical protection/interference studies and gel retardation assays to examine the interactions between Fis and the recombinational enhancer. These studies combined with mutational analysis defined the enhancer sequences required for Fis binding and function. Fis binds with different affinities to two domains within the enhancer sequence. The binding of Fis at each domain is independent of the occupancy of the other domain and appears to be to opposite faces of the DNA helix. These results support a model for the role of the recombinational enhancer in Hin-mediated inversion in which the interaction between Hin bound at recombination sites and Fis bound to each domain of the recombinational enhancer results in a structure with the proper alignment and topology to promote DNA inversion.
\end{abstract}

[Key Words: Recombinational enhancer; site-specific recombination; protein-DNA interactions]

Received June 15, 1987; revised version accepted August 19, 1987.

The observation that a cis-acting DNA sequence can be separated from its site of action by long distances has been made in many different biological systems, but is not yet understood at the molecular level (for reviews, see Dynan and Tjian 1985; Ptashne 1986). The Hin sitespecific recombination system found in Salmonella typhimurium provides a simple model for understanding this phenomenon. The Hin protein mediates a site-specific DNA inversion that is promoted by the presence of a DNA sequence, a recombinational enhancer, which acts in an orientation- and distance-independent manner (Johnson and Simon 1985). Elucidation of the mechanism of action of the recombinational enhancer may help our understanding of the function of the large variety of enhancer sequences found in other systems.

Inversion of the 996-bp DNA segment by Hin alters the expression of flagellin type in Salmonella (Zieg et al. 1977). The recombination reaction requires three DNA sequences: two recombination sites, hix L and hixR, which mark the boundary of the inversion sequence (Zieg and Simon 1980), and the recombinational enhancer (Johnson et al. 1986). Each recombination site is composed of two half-sites with approximate dyad symmetry. The hix sites share a common sequence which must be in inverted configuration for recombination to

${ }^{3}$ Current address: Laboratory of Molecular Biology, National Institute of Mental Health, Building 36, Room 1B08, Bethesda, Maryland 20205 USA. occur. The presence of the recombinational enhancer stimulates the reaction more then 150-fold. Its wild-type location is within the coding region of hin; however, this sequence functions in any location on the substrate plasmid, provided that it is not too close to one of the hix sites (Johnson and Simon 1985). Previous experiments have indicated that the enhancer is composed of two functional domains. The outer boundary of each domain was located by deletion analysis. The existence of two domains was implied by the activity of enhancers containing insertions within the essential DNA sequence (Johnson and Simon 1985).

The Hin protein has been overproduced and purified in E. coli from the cloned gene (Johnson et al. 1986). Hin has a molecular weight of 21,000 and has been shown to bind to both hixL and hix R (Bruist et al. 1984; Johnson et al. 1984). In addition to the Hin protein, two host proteins, Fis (Factor II) and HU, are involved in Hin-mediated inversion. Both proteins have been purified to homogeneity (Johnson et al. 1986). Fis was independently isolated as an approximately 12,000 molecular weight protein essential for recombination in the closely related Gin system of bacteriophage $\mathrm{Mu}$ (see below; Kahmann et al. 1985). Fis and Factor II substitute for one another and are assumed to be the same protein (R. Kahmann et al. and R. Johnson, unpubl.). Fis has been demonstrated to bind to the Gin enhancer DNA (Koch and Kahmann 1986). The details of the interaction between Fis and the 
Hin recombinational enhancer are presented in this report.

The requirement for HU varies with the substrate. There is a strong stimulation of recombination by $\mathrm{HU}$ for the wild-type substrate and other substrates in which the recombinational enhancer is near $(<350 \mathrm{bp})$ to one of the crossover sites. However, in reconstructed substrates where there is a large distance from the enhancer to either crossover site, there is almost full recombination activity in the absence of HU. Thus, HU may help looping of the DNA to allow interaction between Hin bound at the hix recombination sites and Fis bound at the enhancer sites (Johnson et al. 1986).

Hin is a member of a family of site-specific recombinases which includes among others Gin and Cin. Deletion analysis has shown that both gin and cin contain recombinational enhancers in the $5^{\prime}$ region of the coding sequence in analogous positions to the enhancer in the hin gene (Huber et al. 1985; Kahmann et al. 1985). To define clearly the nature of the Hin enhancer sequence, the Fis binding sites, and the mechanism of action of the enhancer, we undertook the nuclease and chemical protection/interference experiments and gel binding studies described in this report.

\section{Results}

DNase I cleavage protection indicates that Fis binds to two domains of the recombinational enhancer

Our results indicate that Fis binds to two sites within the recombinational enhancer region. These sites are dissimilar in base sequence and are designated the proximal and distal domains, based on their distance from hix $L$ in the ON orientation (see Fig. 1). The binding was initially demonstrated using DNase $I_{;}$its ability to cleave at specific sites is blocked or enhanced by the presence of a bound protein (Galas and Schmitz 1978). The first panel in Figure 2 shows the DNase I protection pattern produced by Fis on the noncoding strand of the wild-type enhancer region from the plasmid pMS551. The total protected region at the distal domain is about 25 bases long and at the proximal domain it is slightly shorter. Each protected region is interrupted twice by short unprotected regions ( $2-3$ bases) marked by single enhanced cleavages that are 12 or 13 bases apart. A similar DNase I protection pattern is observed for the upper strand with the DNase I cleavage enhancements shifted by two or three bases in the 3 ' direction relative to the bottom strand. The results are summarized in Figure 3. There is no evidence for Fis-DNA interactions in the region between the two domains.

The DNase I protections in the enhancer region are dependent only on the presence of Fis. Protections in this region have been detected using whole-cell lysates (Johnson et al. 1984) and using partially purified preparations of the Hin protein (Johnson and Simon 1985). Purer fractions of the Hin protein (approximately $90 \%$ pure; Johnson et al. 1986), which require the addition of Fis for inversion activity, do not show protections in this region. The presence of HU, Hin, and/or Hin binding sequences do not influence the DNase I cleavage protections by Fis at the enhancer on either linear fragments or supercoiled DNA substrates (A.C. Glasgow, M.F. Bruist, and M.I. Simon, in prep.). Fis gives a similar DNase I cleavage protection pattern on enhancers that have different DNA sequences surrounding them.

DNase I cleavage protection patterns of the enhancer with increasing concentrations of Fis (data not shown) indicated that the distal domain is protected by lower Fis concentrations than the proximal domain. At protein concentrations only slightly higher than that necessary to protect the proximal domain, several additional protected sequences are evident. Many of these protections are distinguished by a characteristic pair of enhancements 12-14 bp apart, similar to those seen in the enhancer domains (data not shown). None of these additional Fis binding sites is required for recombination

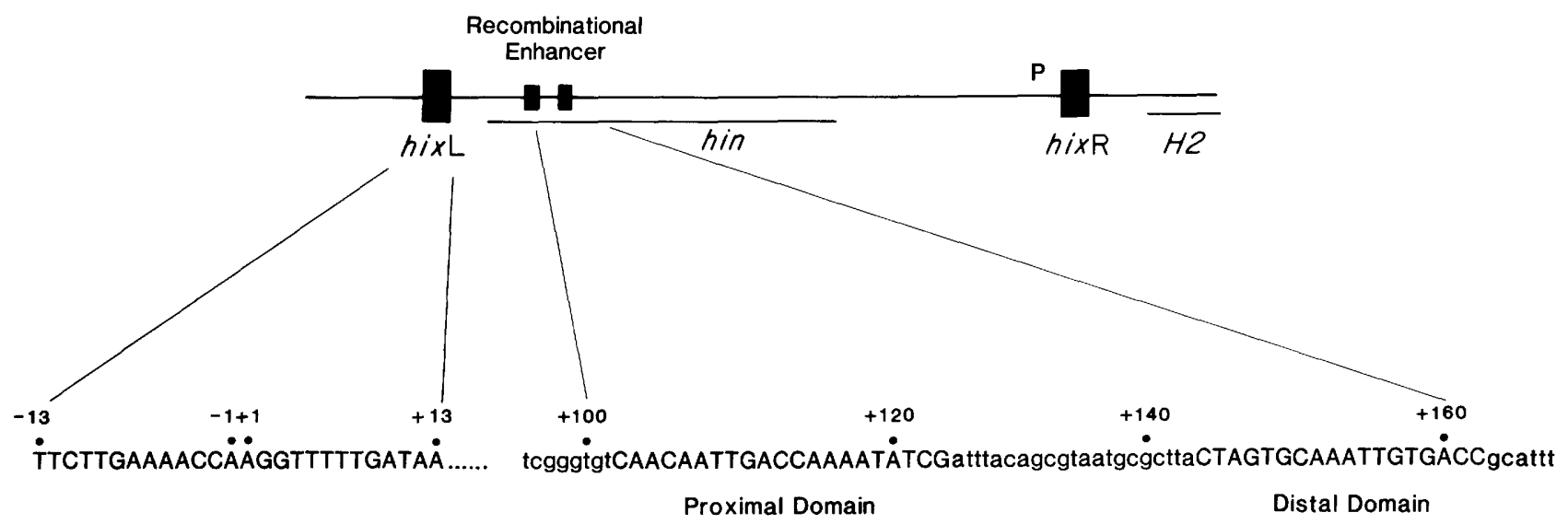

Figure 1. Diagram of the invertible DNA segment controlling flagellin expression in Salmonella. Depicted is the ON orientation of the invertible Hin segment in which the promoter $\langle\mathrm{P}\rangle$ is in the proper orientation for transcribing the H2 flagellin gene. The approximate positions of the recombination sites, hix $\mathrm{L}$ and hixR, and the recombinational enhancer are shown. The sequences for the hixL site and for the enhancer are given. The proximal and distal domains of the enhancer are indicated by the upper-case letters. 


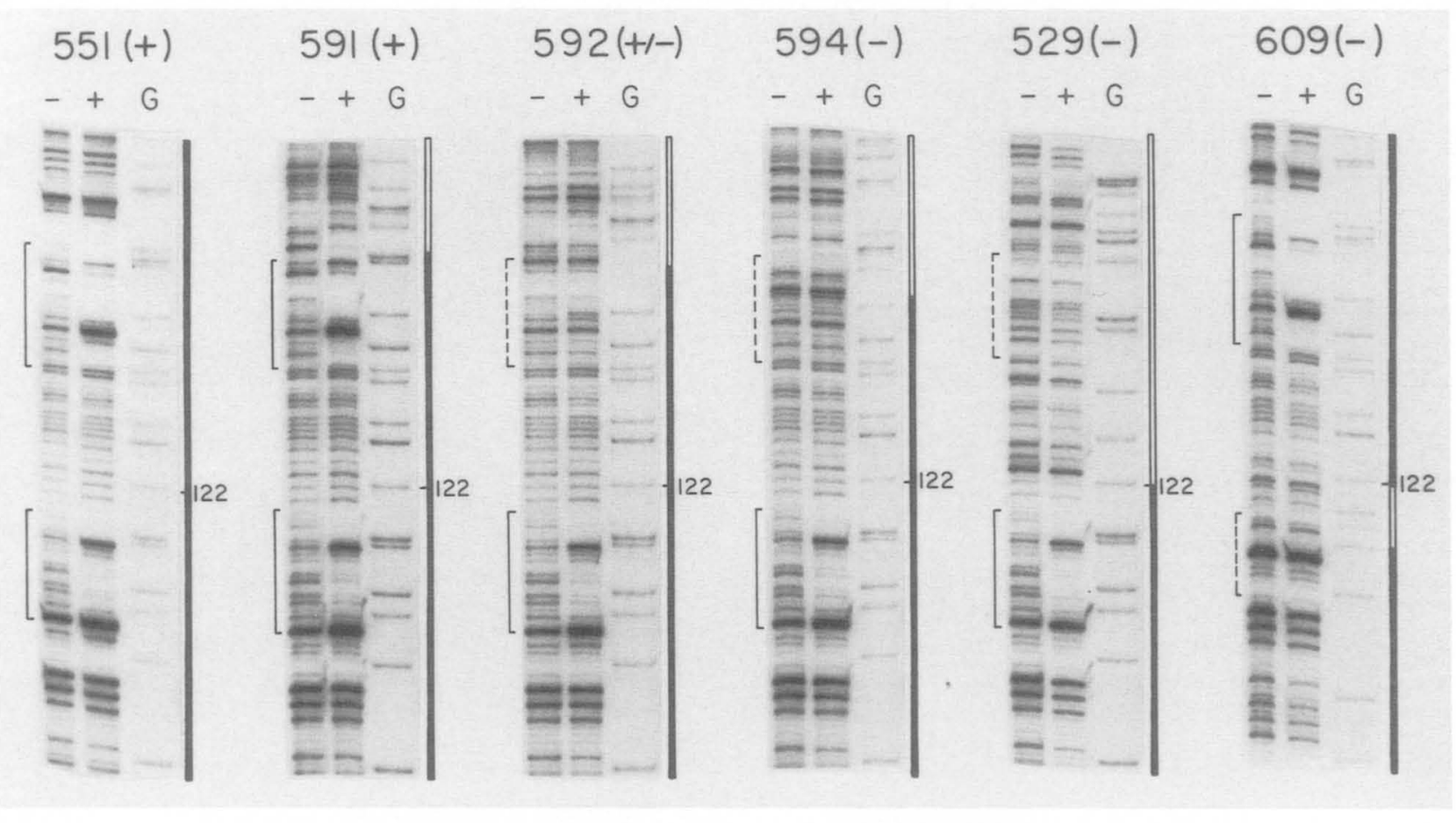

Figure 2. Fis binding to a series of enhancer deletions. The DNase I cleavage protection pattern given by Fis is shown for a series of EcoRI-NruI restriction fragments from plasmids pMS551, pMS591, pMS592, pMS594, pMS529, and pMS609 which were 3'-end-labeled with $\left[\alpha^{32} \mathrm{P}\right] \mathrm{dATP}$ at the EcoRI site. In each panel the first lane contains the guanine sequence reaction to indicate the sequence of the fragment. The second lane $(-)$ is the control with no protein added and the third lane $(+)$ shows the protection given by $250 \mathrm{ng}$ of partially purified E. coli extract containing Fis and Hin (Johnson and Simon 1985). Only the section of the gel displaying the enhancer sequence is shown. The DNase I reactions were carried out as described in Materials and methods. The location of the enhancer domains are indicated by brackets on the left of each set of lanes, the deletions and substitutions are indicated on the right where the open line indicates nonenhancer DNA. The relative recombination activity of each plasmid substrate is indicated in parentheses above each set of lanes (see also Table 1). Base 122 is indicated in the cleavage pattern as a reference.

since they can be deleted (Johnson and Simon 1985); however, they may explain the weak stimulation by Fis on recombination in the absence of a wild-type enhancer (Johnson et al. 1986). Fis may be able to recruit a pair of these secondary sites to function as an enhancer.

\section{Gel retardation assays and chemical interference studies distinguish binding to each of the domains}

The gel retardation assay, which separates discrete protein-DNA complexes by electrophoresis in nondena-

Proximal Domain turing polyacrylamide gels (Garner and Revzin 1981; Fried and Crothers 1981), was used to characterize further the binding of Fis to the enhancer. Figure 4A shows the formation of such complexes with a DNA fragment, containing the enhancer, as a function of increasing concentration of Fis. Two discrete bands are observed migrating above the protein-free DNA fragment, corresponding to complexes having one (complex 1) and two (complex 2) domains of the enhancer occupied by Fis. Complex 1 has Fis bound predominantly to the distal domain, while complex 2 has both the distal and prox-

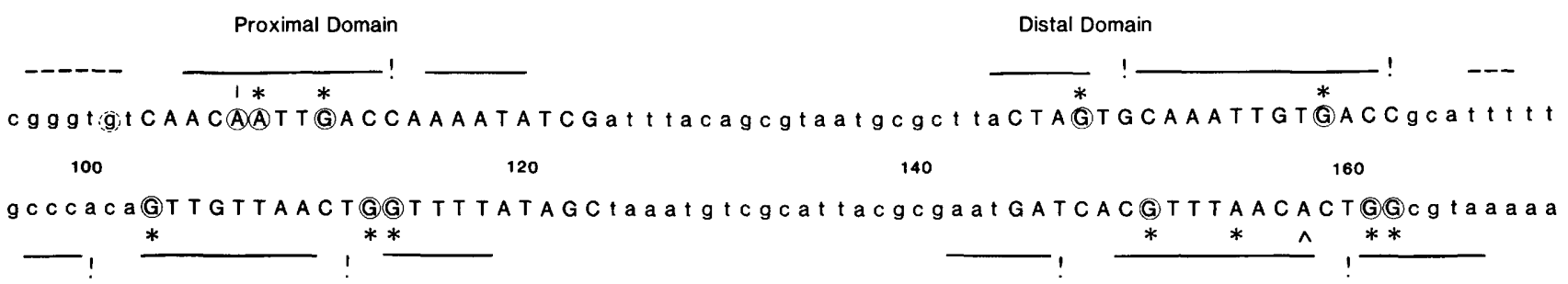

Figure 3. Summary of DNase I and methylation protection/interference experiments. The sequence of the region containing the recombinational enhancer is shown from base pairs +96 to +170 . The large letters within the enhancer sequence indicate the approximate location of the proximal and distal domains of the enhancer as defined by deletion and substitution analyses. DNase I-protected bases are indicated by solid lines above and below the sequence; partial protection is indicated by dashed lines. The bases showing DNase I-enhanced cleavage are marked by exclamation points. Purines protected from methylation are symbolized by asterisks; partially protected bases are marked with a vertical bar and weak methylation enhancements are indicated by a single caret. Base contacts determined by methylation interference experiments are denoted by circled residues. Dotted circles represent partial interference. 
A

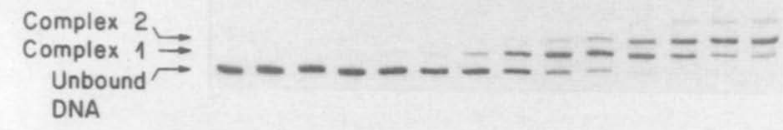

B

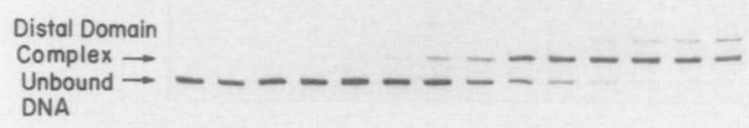

C

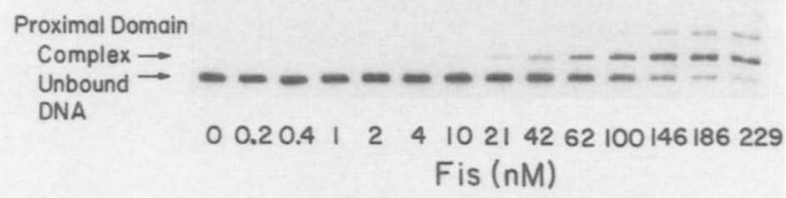

D

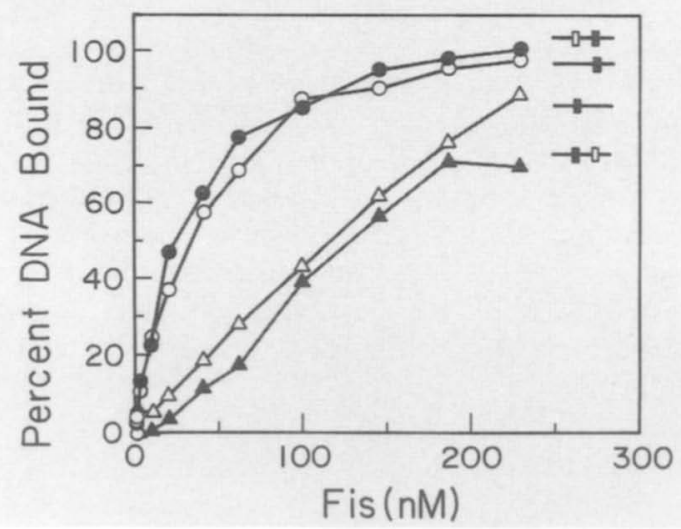

Figure 4. Titrations of Fis binding to the recombinational enhancer and each isolated domain. Increasing concentrations of purified Fis (indicated below gels) were incubated with end-labeled fragments from pMS551 (wild type), pMS609 (deletion of proximal domain), and pMS529 (deletion of distal domain) and the Fis-DNA complexes were separated by gel electrophoresis as described in Materials and methods. The autoradiogram in $A$ shows the binding of Fis to the wild-type enhancer; complex 1 has Fis bound to the distal domain and complex 2 has Fis bound to both domains (complexed and unbound DNA are indicated by arrows). ( $B$ and $C$ ) Fis binding to the isolated distal (pMS609) and proximal (pMS529) domains, respectively. The unbound DNA and the primary complex, where either the distal or proximal domain is bound by Fis, are indicated by arrows. Additional slower-migrating bands are presumed to have secondary sites occupied by Fis. $(D)$ Plot of the binding data derived from the titrations shown in $A, B$, and $C$. Percent DNA bound was calculated as described in Materials and methods. Closed symbols represent titration data for the wild-type enhancer and open symbols indicate the deleted derivatives. Circles denote binding to the distal domain and triangles depict the proximal domain binding. Illustrated to the right of each plot is the domain (solid box) whose binding is represented by the adjacent curve.

imal domains occupied (see below). At higher concentrations of Fis $(>100 \mathrm{nM})$ the secondary sites become occupied, giving rise to the slower-migrating complexes observed. Complexes 1 and 2 are resistant to the addition of heparin or a large molar excess of nonspecific DNA (calf thymus or pBR322), while the formation of the slower-migrating complexes is sensitive to the addition of these competitors.

To confirm the location of the sites occupied in complexes 1 and 2 and to determine the important contacts made by Fis in each enhancer domain, chemical interference of binding experiments (Siebenlist and Gilbert 1980) were performed as modified by Hendrickson and Schleif (1985). The DNA was partially methylated with dimethyl sulfate (DMS) and then incubated with Fis protein and electrophoresed in a native polyacrylamide gel to separate the unbound DNA and the various proteinDNA complexes. DMS methylates at the $\mathrm{N}-7$ position of guanine in the major groove and the $\mathrm{N}-3$ position of adenine in the minor groove. DNA fragments methylated at adenines or guanines that are important for proper protein-DNA recognition and contact will not be found in a protein-DNA complex. The purine contacts in the occupied sites on the DNA are identified on a DNA sequencing gel as uncleaved residues from the isolated complex DNA after chemical cleavage of the methylated residue.

The results of this experiment for the coding and noncoding strands of the full enhancer sequence are shown in Figure 5. As shown in lanes 2 and 6 the distal domain of the enhancer is occupied in complex 1 , and both domains are occupied in complex 2 (lanes 3 and 7). The methylated DNA from the isolated unbound fraction shown in lane 5 is enriched with fragments cleaved at residues critical for Fis binding to both the distal and proximal domains. There are also some enhanced residues from the proximal domain seen in the complex 1 fraction, since these methylated bases would interfere with formation of complex 2 . The partially methylated bases within the proximal domain (lanes 3 and 7) can be attributed to some doubly occupied complexes having Fis bound to the distal domain and one secondary site. The important contacts determined for both strands in each domain are summarized in Figure 3. These results are consistent with the DNase I studies and confirm that the distal domain is the first domain of the enhancer to be occupied.

Figure 3 also summarizes the results of methylation protection studies, in which the presence of a bound protein can block or enhance the ability of DMS to methylate either guanine or adenosine (Gilbert et al. 1976). All purines identified as close contacts by methylation interference were also found to be protected from methylation. There are no differences in the contacts made by Fis within the distal domain regardless of the occupancy of the proximal domain.

\section{Binding of Fis to isolated proximal and distal domains}

DNase I cleavage protection studies of Fis binding to substrates deleted for the distal or proximal domain (pMS529 and pMS609, respectively) show that the protection pattern is the same for each isolated domain as for both domains in the wild-type enhancer (see Fig. 2). Gel binding experiments using these modified enhancers, pMS529 and pMS609, are shown in Figure 4, B and $\mathrm{C}$. These titrations demonstrate that binding occurs to each independent domain with affinities similar to 


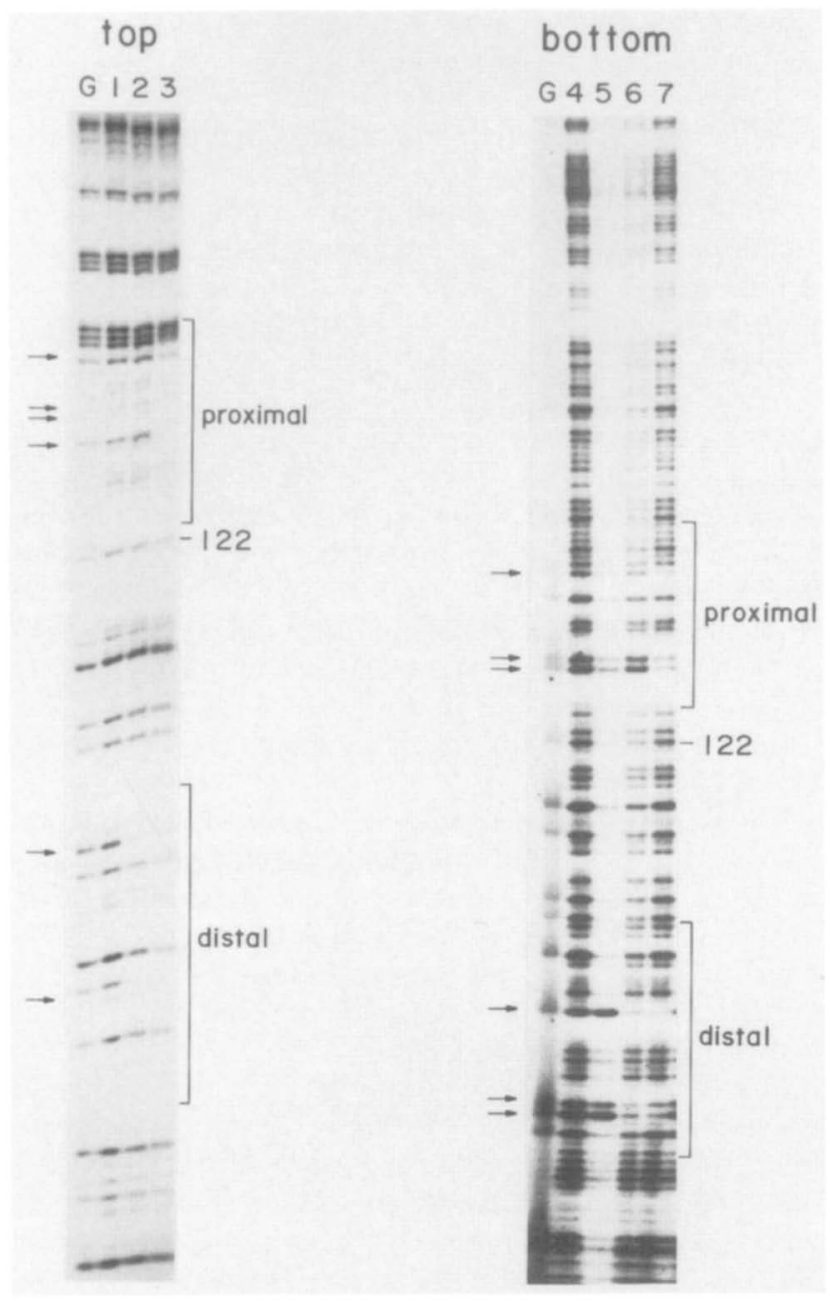

Figure 5. Methylation interference assays. Cleavage products from methylation interference assays for the coding (top) strand and noncoding (bottom) strand of the recombinational enhancer are shown here on DNA sequencing gels (see Materials and methods). (Lanes 1 and 4) Minus Fis; (lanes 2 and 6) complex 1; (lanes 3 and 7) complex 2. (Lane 5) Unbound DNA fraction which shows enhanced bands at the purine residues whose methylation prevents binding at either domain; (lanes G) DNA sequencing reactions for guanine residues. The arrows indicate critical contacts in the proximal and distal domains (bracketed on the right of each gel). Base 122 is marked in the cleavage pattern of each strand as a reference.

the intact enhancer and that the isolated distal domain binds Fis stronger than the proximal domain. The binding of Fis in these experiments was measured by determining the mole fraction of DNA in each discrete complex as described in Materials and methods. Figure 4D shows a plot of the percent DNA bound by Fis at the proximal and distal domains versus the total concentration of added Fis for titrations of the wild type (pMS551) and deleted enhancer substrates. These results demonstrate that the binding affinity of Fis for the enhancer domains is the same whether the domains are isolated or in the intact enhancer. Thus, there is no evidence for cooperativity that facilitates binding of Fis at the two domains of the enhancer. The apparent binding con- stants for the distal and proximal domains, based on the Fis concentration required for $50 \%$ occupancy of each domain, are approximately $24 \mathrm{nM}$ and $120 \mathrm{nM}$, respectively. These are minimal values for the dissociation constants since the concentration of active Fis may be lower than the determined protein concentration.

\section{Fis binding to mutant enhancers}

DNase I cleavage protection studies of various deletion and substitution mutants of the enhancer were performed to define the boundaries of the distal domain. A deletion extending into the right boundary of this domain through base pair +164 (pMS591), affected neither binding (Fig. 2) nor activity of the enhancer (Table 1). Deletion of the next four bases into the distal domain (pMS592) diminishes both enhancement activity (Table 1) and binding. As seen in Figure 2, a standard concentration of Fis gives no detectable protections throughout the entire distal domain of pMS592 and only slight cleavage enhancement at bases $\mathrm{C}+150$ and $\mathrm{C}+159$ (bottom strand, see Fig. 3). The rate of recombination of pMS592 is approximately threefold below the recombination rate of the wild-type substrate, but still more than 10-fold above an enhancer-impaired substrate (pMS594). Thus, it appears that the four additional bases deleted in pMS592 relative to pMS591 are important for higher binding affinity of Fis to the distal domain, but deletion of these bases does not completely eliminate binding or enhancer function. A deletion of seven more bases (pMS594) completely eliminated the enhancer function and Fis gave no protections or enhancements of DNase I cleavage. For all of these distal domain deletions (pMS591, pMS592, and pMS594) the binding to the proximal domain was normal. Thus, the enhancer domains behave as independent Fis binding sites.

The left boundary of the distal domain was indicated by the binding affinity of Fis for two mutants: pMS640 which has a 4-bp insertion between +144 and +145 and pMS641 which has a 1-bp insertion between +147 and +148 (see Table 1). Fis has only a slightly (approximately twofold) reduced affinity for the distal domain of pMS640 as compared to the wild-type sequence; however, pMS641 is significantly (approximately 10-fold) reduced in its ability to bind Fis as measured by titrations in gel binding assays and DNase I cleavage protection studies (data not shown). Therefore, the sequence between +144 and +148 is necessary for maximal binding affinity of Fis to the distal domain. The difference in binding affinity to Fis for the distal domain in pMS640 and pMS641 is not accurately reflected in the inversion rates of these mutants given in Table 1 due to the changes in spacing between the two domains. The precise spatial relationship of the proximal and distal domains has been shown to be critical for enhancer function (Johnson et al. 1987).

\section{A mutant enhancer that defines the minimal Fis binding sequence}

A plasmid (pMS710) was constructed in which the proximal domain of the enhancer was substituted by a syn- 
Table 1. Inversion rates for various mutant substrates

\begin{tabular}{|c|c|c|}
\hline Substrate & & Inversion rate \\
\hline & 160 & \\
\hline pMS551 & ...CTAGTGCAAATTGTGACCGCATTT. . . & 0.62 \\
\hline pMS589 & ...CTAGTGCAAATTGTGACCGCg g a a . . . & 0.58 \\
\hline pMS591 & ...CTAGTGCAAATTGTGACCGg a a $\mathrm{g} \mathrm{ct} \ldots$ & 0.65 \\
\hline pMS592 & ...CTAGTGCAAATTGTGg g a a $\mathrm{c} t \mathrm{t} \mathrm{c} \ldots$ & 0.23 \\
\hline pMS594 & ...CTAGTGCAg g a g $\mathrm{ct} \mathrm{t} \mathrm{c} .$. & $<0.02$ \\
\hline pMS640 & ...c t a g CTAGTGCAAATTGTGACCGCATT & $<0.02$ \\
\hline pMS641 & ...c t a g g TGCAAATTGTGACCGCATTT. . & 0.29 \\
\hline
\end{tabular}

Inversion rates (inversions per molecule) were measured as described (Johnson et al. 1986). The inversion rates for pMS640 and pMS641 reflect the altered spacing between the proximal and distal domains due to their insertions of 4 and 1 bp, respectively (Johnson et al. 1987) as well as changes to the distal domain sequence. The small letters indicate the altered sequence in the deletion and substitution mutants; the positions of the nucleotides are shifted for pMS640 and pMS641 to reflect the changes in spacing between domains created by the insertions. The numbers marked above the sequence indicate the position of these bases within the enhancer sequence.

thetically derived sequence that includes nucleotides +150 to +162 of the distal domain (Fig. 6B). The novel proximal domain in pMS710 contains the bases thought to be important for Fis binding based on DMS binding interference experiments, analysis of deletion mutants, and a comparison of related enhancer sequences from the homologous DNA recombinases (see Discussion). The spacing between the proximal and distal domains in pMS710 is identical to the wild-type enhancer based on the locations of these proposed recognition sequences (see Fig. 6B). DNase I cleavage protection of pMS710 (Fig. 6C) indicates that Fis binds to the synthetically de-
A.

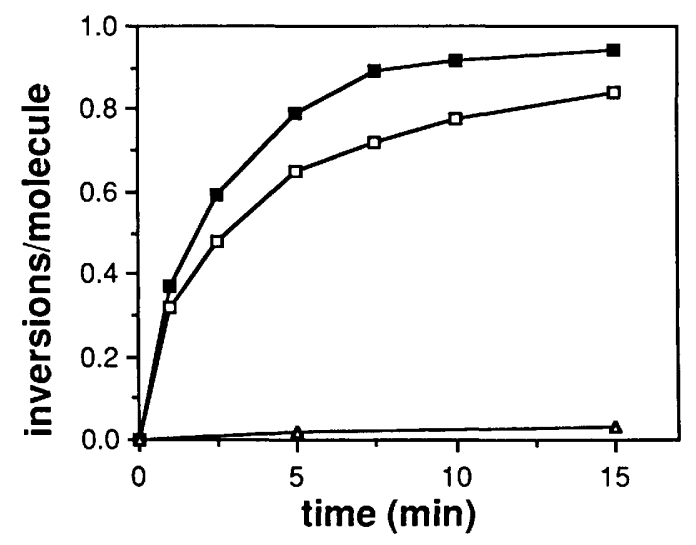

B.

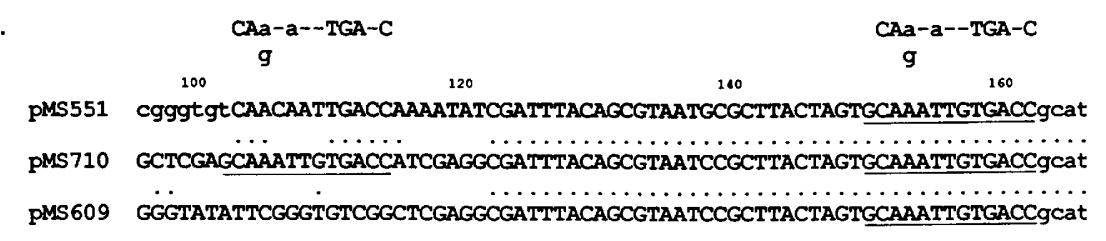

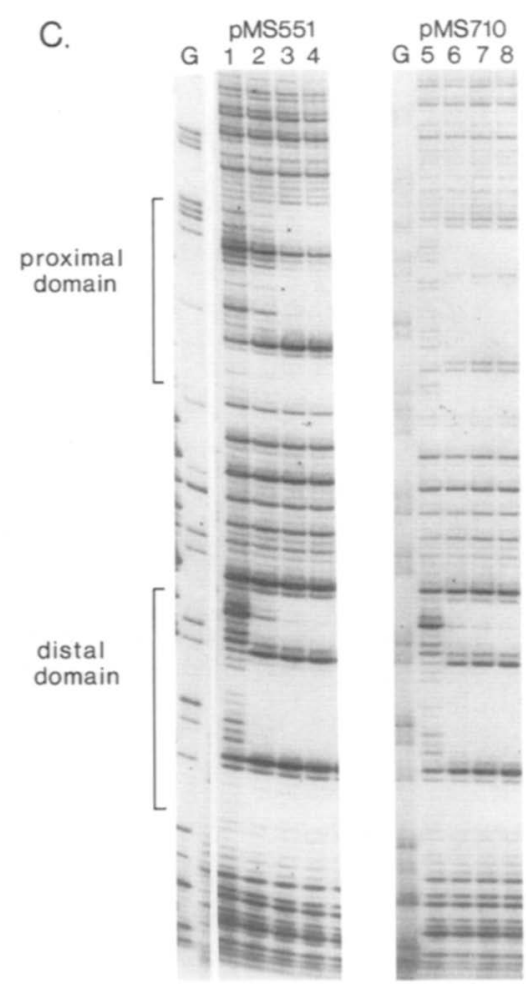

Figure 6. Activity and binding of a synthetically derived enhancer mutant. (A) Time course for the inversion activity of pMS710 (open squares) which contains a synthetically derived proximal domain and wild-type distal domain, and compares it to the inversion time course for a substrate containing the wild-type enhancer, pMS551 (a), and a mutant substrate which is deleted for the proximal domain, pMS609 $(\Delta)$. The enhancer sequence for each of these substrates is shown in $B$. The underlined sequence indicates the conserved distal domain sequence that was substituted for the proximal domain in pMS710; the dots denote conserved bases between the wild-type enhancer and the mutant enhancers pMS710 and pMS609. $(C)$ DNase I cleavage protections by purified Fis (Johnson et al. 1986) on the coding strands of the wild-type enhancer (lanes 1-4) and pMS710 (lanes 5-8). Lanes 1 and 5 have no added Fis; lanes 2 , 3,4 and 6, 7, 8 have increasing concentrations of Fis added (21 nM, $62 \mathrm{nM}$, and $125 \mathrm{nM}$ Fis). (Lanes G) Chemical cleavage of guanines in each case. 
rived proximal domain in a manner similar to the distal domain. The total protected area spans approximately 22 bp with two regions of enhancements and unprotected bases at 12-bp intervals. Titrations of Fis binding using both DNase I cleavage protection (Fig. $6 \mathrm{C}$ ) and gel retardation assays (data not shown) demonstrated that the binding affinity for the proximal domain of pMS710 was within twofold of the wild-type distal domain. In Figure $6 \mathrm{~A}$, the recombination activity of pMS710, pMS551 (wild type), and pMS609 (deleted for the proximal domain) are shov'n. pMS710 recombines at a rate that is essentially identical to the wild-type, indicating that the enhancer is fully functional.

\section{Discussion}

Fis interacts independently with two domains of the recombinational enhancer

Site-specific recombination in the Hin system is stimulated up to 150-fold by the presence of a DNA sequence called the recombinational enhancer. We have characterized the binding of Fis, a protein essential for recombination, to the recombinational enhancer using DNase I and DMS cleavage protection and DNA gel retardation assays. The enhancer consists of two domains, called proximal and distal, each of which is independently bound by Fis. The region protected from DNase I at each domain is about $24 \mathrm{bp}$ in length and is interrupted twice. The protections at each domain extend beyond the approximate boundaries determined by the deletion and modification experiments presented herein and by Johnson and Simon (1985) (see Fig. 3). This probably reflects the size of the DNase I binding site and possible nonspecific interaction of Fis with DNA beyond its specific recognition sequence. It has been reported (Suck and Oefner 1986) that DNase I covers 4 bases $5^{\prime}$ and 6 bases $3^{\prime}$ to a cut on a given strand. With these limits the boundaries of Fis on DNA as seen by DNase I agree with the deletion analysis.

The results of these DNase I protection studies and the methylation interference experiments indicate that Fis spans two major grooves, making contacts in the major and minor grooves. The disposition of the purine contacts suggests that Fis binding at each domain is primarily to one side of the DNA helix. It has not been determined whether Fis binds to the enhancer as a monomer or as a higher multimer. Koch and Kahmann (1986) have shown that Fis exists in solution predominantly as a dimer and that the dimer was the active species for binding the Gin enhancer. Protection of a partial domain was not observed in our experiments, indicating either that Fis monomers bind very cooperatively or that Fis is only active for binding as a multimer.

\section{The nature of the sequence recognized by Fis}

Comparison of the sequence in the regions of the proximal and distal domains revealed two shared pentanucleotide sequences, TGACC and AATTG. Either se- quence could be part of a recognition sequence for Fis binding, but since the spacing of these sequences is different within the two domains (they overlap in the proximal domain|, it is unlikely that both are a part of the same recognition sequence. In each domain methylation of any of the guanines in the double-stranded sequence specified by TGACC interferes with binding and Fis induces enhanced DNase I cleavage at one of the two cytidines. Thus, Fis is probably in the same position relative to this sequence in each domain, indicating that this sequence is likely to be a part of a recognition sequence.

The two TGACC sequences are $48 \mathrm{bp}$ apart and therefore separated by about 4.5 turns of the double helix. The finding that the mutant pMS710, which is composed of two copies of the distal domain in direct repeat configuration separated by this spacing, supports wild-type levels of recombination confirms the fact that the two domains are separated by $48 \mathrm{bp}$. Other experiments (Johnson et al. 1987) have shown that the relative disposition of the domains is critical for enhancer function. Therefore, it appears that the two Fis molecules must sit on opposite faces of the DNA to function in stimulating Hin-mediated inversion.

Table 2 compares the DNA sequence of the domains of the enhancers for the homologous inversion systems, Hin, Gin, and Cin. The most conserved bases suggest the consensus sequence CAPu.a..TGA.C for Fis recognition. It should be noted that the enhancers are part of the translated portion of the recombinase genes and that some of the conserved bases could reflect the need for conservation of amino acids in the proteins, rather than conformation to a Fis recognition sequence. This problem does not exist when comparisons are made between distal homologous and proximal homologous domains. A binding site for Fis near the Xis binding site in bacteriophage $\lambda$ has been described recently (Thompson et al. 1987) and does not appear to have this sequence. It is possible that Fis recognizes a particular structural feature of the DNA rather than, or in addition to, a specific recognition sequence as suggested for the interaction of HAP I with the activation sites of $\mathrm{CYCl}$ and $\mathrm{CYC7}$ (Pfeifer et al. 1987). Further studies are necessary to determine the DNA sequence or structural features involved in Fis recognition of the recombinational enhancer.

The sequence substituted for the proximal domain in pMS710 includes bases +150 through +163 which contains the proposed consensus sequence. The high rate of recombination for pMS710 and the nearly wild-type binding affinity of Fis for the synthetically derived proximal domain suggests these nucleotides constitute a minimal sequence for a functional domain. However, it should be noted that immediately upstream of the proximal domain of pMS710 is the sequence CTNGN which would correspond to nucleotides +145 through +149 of the distal domain. Thus, this mutant proximal domain may actually utilize important positions (e.g., at G-148) in this short upstream sequence and therefore have boundaries consistent with those of the distal domain defined by insertional analysis $(+145$ through +162$)$. 
Table 2. Comparison of the nucleotide sequence of the various domains of the recombinational enhancers of the Hin family of recombinases ${ }^{\mathrm{a}}$

\begin{tabular}{|c|c|}
\hline $\begin{array}{l}\text { taCTAGTGCAAATTGTGACCg c a t } \mathrm{t} \\
\text { tgTTTGTGCAGGATGTGAACa a a t a } \\
\text { tgAAAGCGCAGGATGTGAGCt a a t } \mathrm{t} \\
\text { tcg g g t g t CAACAATTGACCAAAAT } \\
\text { aa g g t a t CAACAAATGACCAGAAT } \\
\text { acg c g t a t CAACAAATGAACAAAAC } \\
\text {. . . . CA } \text {. a . TGA. C. }\end{array}$ & $\begin{array}{l}\text { Hin distal domain } \\
\text { Gin second domain } \\
\text { Cin second domain homologd } \\
\text { Hin proximal domain } \\
\text { Gin first domain } \\
\text { Cin first domain homologd } \\
\text { Consensus }\end{array}$ \\
\hline
\end{tabular}

a Recombinational enhancers have been grouped by their positional homology to the hin distal and proximal domains. The alignment of the sequences between enhancers from different recombination systems is that which gives the maximum homology of the entire gene sequence. The approximate location of the enhancer domains for Gin and Cin have been defined by deletion analyses /Huber et al. 1985; Kahmann et al. 1985). DNase I cleavage protections for Fis binding to the Gin enhancer indicate a third domain (Kahmann et al. 1987); however, only slight homology to the Hin distal or proximal domains was found. The distal and proximal domains have been aligned on the sequence TGACC for the reasons outlined in the discussion. Bases indicated by capital letters are homologous to bases within the hin enhancer domains needed for full activity. Underlined bases are in base pairs which are altered in DMS reactivity by Fis.

b Zieg and Simon (1980).

c Plasterk et al. (1983).

d Hiestand-Nauser et al. (1983).

${ }^{e}$ The consensus sequence considered only those bases needed for full activity. Lower-case letters indicate positions where there may be a degenerate consensus.

\section{Proposed model for the role of Fis in Hin-mediated DNA inversion}

Figure 7 presents a model for the possible mechanism of Hin-mediated recombination. This model is closely related to the two-step synapsis model for resolvase-mediated recombination presented by Boocock et al. (1986, 1987). The resolvases found in the transposons gammadelta or Tn3 is distantly related to the Hin family of re- combinases, sharing $30-40 \%$ amino acid sequence homology with these recombinases; but it does not complement any of their activities (Simon et al. 1980). Resolvase promotes recombination between two directly repeated res sequences on a transpositional intermediate to give two independent replicons and does not require any additional DNA sequences, such as a recombinational enhancer, or other protein factors for function (Reed 1981). However, the res sites themselves are com-

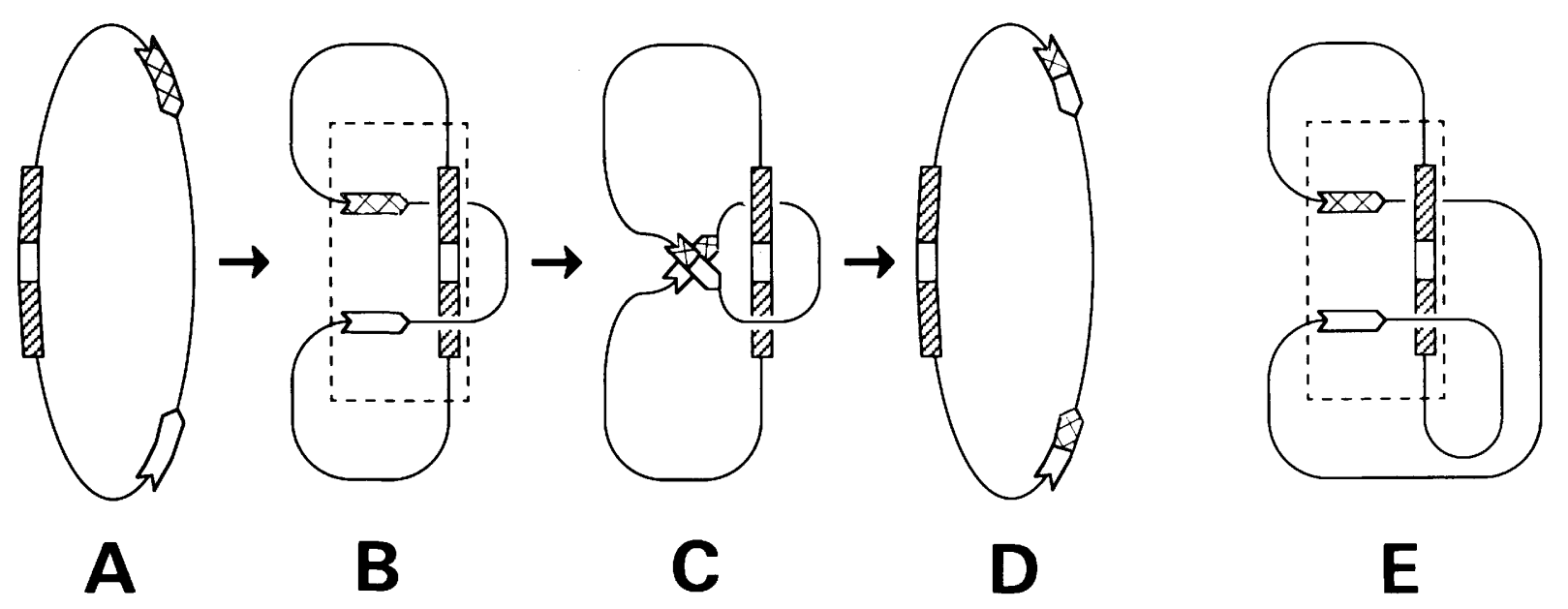

Figure 7. A model for the role of the recombinational enhancer in Hin-mediated site-specific recombination. The inversion of a segment of DNA on a plasmid is illustrated. For simplicity only the DNA is shown and it is depicted as a relaxed circle even though supercoiling is required. Two inverted repeat recombination $($ hix $)$ sites are indicated by arrows, one of which has been cross-hatched, and the recombinational enhancer is indicated as two connected, shaded domains. Synapsis of the hix sites and the enhancer domains in substrate $A$ that produces the structure boxed in $B$ will give rise to recombination. This structure may be determined by contacts between Hin and Fis (not shown) bound to their respective DNA sites. The two negative nodes in $B$ will be stabilized by negatively supercoiled DNA. When the strand exchange proceeds with the polarity indicated in $C$, the resulting product $D$ will be an unknotted circle with the DNA between the two recombination sites inverted. The strand exchange reverses the sign of the two negative nodes in $B$, introduces a new negative node in the product, and mechanistically introduces a twist of +1 . Thus, there is a net change of +4 in the linking number of the plasmid (for a detailed explanation see Cozzarelli et al. 1984). A synaptic structure similar to that shown in $B$ can be made on a plasmid containing directly repeated recombination sites $(E)$; however, the nodes in this structure are of opposite sign and will not be stabilized by the supercoiling. This may explain the preference of Hin for acting on inverted repeats. 
plex as they contain three subsites, only one of which recombines (Grindley et al. 1982). Boocock et al. (1986, 1987) have proposed a model for the mechanism of action of resolvase in which the two additional subsites (sites II and III) within each res site serve to align properly the res sites at site I where strand exchange occurs. In this model specific nodes must be created by the DNA strands at the crossover sites; this gives rise to the topological specificity of the reaction (see also Craigie and Mizuuchi 1986; Gellert and Nash 1987).

The Hin model is based on the assumption that Hin, Fis, and their respective DNA binding sequences must form an intermediate complex containing defined DNA crossover nodes for inversion to occur (see the boxed structure in Fig. 7B). The nodes may be formed by interactions between Fis and Hin bound to their respective sites and the sign of these nodes is determined by the location of the Fis molecules which are bound to opposite sides of the DNA helix, as predicted by the binding studies. Therefore, the two domains of the enhancer perform a role analogous to that proposed for subsites II and III of the res site of the resolvase system. As drawn, the two enhancer nodes have the same configuration as the two nodes nearest the crossover point for the proposed resolvase intermediate (see Boocock et al. 1987). Both nodes are negative and therefore the structure will be stabilized by negative supercoiling. The nodes determine three loops of DNA which can be of any length. The freedom in the size of these loops accounts for the ability of the enhancer to act at many different locations. The required bending of the DNA /approximately $\left.270^{\circ}\right)$ to form this node agrees with the explanation offered by Johnson et al. (1986) for how HU stimulates recombination when the enhancer is located $<350$ bp from the recombination site. When the enhancer is located within $30 \mathrm{bp}$ from the center of the recombination site it does not stimulate recombination even in the presence of HU. The inability for the enhancer to function at this distance is not the result of steric interference between Hin and Fin proteins bound at their respective sites, as determined by DNase I cleavage protection (M. Bruist, unpubl.), suggesting that the torsional strain of DNA bending is preventing the formation of these loops resulting in the loss of enhancer activity observed (Johnson et al. 1986).

A strand exchange reaction with the sense of that depicted in Figure 7C will invert the DNA between the hix sites and give an unknotted product with four fewer supertwists (Fig. 7D), as explained in the legend for Figure 7 and in Cozzarelli et al. (1984). The change in linking number is in agreement with the observations of others (Kahmann et al. 1987; Kanaar and van de Putte 1987). Furthermore, multiple rounds of recombination without disruption of the nodes indicated in $7 \mathrm{C}$ will produce a knot. Such knots have been observed by Kanaar and van de Putte (1987).

A similar structure can be formed using a substrate containing directly repeated hix sites (Fig. 7E). However, this structure has one positive and one negative node and thus is not stabilized by supercoiling. If conditions for Hin recombination using relaxed substrates are discovered for Hin, as have been found for Int- and resolvase-mediated recombination (Nash and Pollock 1983; Boocock et al. 1987), it is predicted that there will no longer be a strong bias against recombination of directly repeated hix sites. A strand exchange reaction with the same sense as depicted in Figure $7 \mathrm{C}$ will generate two unlinked circles from directly repeated crossover sites.

Continued studies to test the proposed model for the enhancer function in Hin-mediated site-specific recombination will provide guidelines for the study of other DNA cis-acting sequences that operate at a distance. Clearly, multiple protein-DNA complexes with strictly defined topologies will be important in many of these phenomena.

\section{Materials and methods}

\section{Plasmid construction}

Most plasmids used in this study have been described in Johnson and Simon (1985). pMS710 was constructed as follows. pMS609, which contains a XhoI linker fragment substituted for the proximal domain (Johnson and Simon 1985), was cleaved with $X$ hoI and the $5^{\prime}$ ends dephosphorylated using calf intestinal phosphatase. A 500-fold molar excess of the synthetic oligonucleotides TCGAGCAAATTGTGACCA and TCGATGGTCACAATTTGC which had been phosphorylated with polynucleotide kinase and ATP were hybridized and ligated with the digested pMS609. DNA sequence analysis (Sanger et al. 1977) of the entire enhancer region in the resulting plasmid (pMS710) confirmed the sequence shown in Figure 6B. pMS640 was constructed by digesting pMS591 (Johnson and Simon 1985) with SpeI which cleaves at position +144 in the enhancer, filling in the protruding ends with T4 DNA polymerase and dNTPs, and ligating the blunt ends with T4 DNA ligase. pMS641 was made in a similar manner except that dTTP was omitted from the polymerization reaction. DNA sequence analysis indicated that pMS640 and pMS641 have the sequence given in Table 1.

\section{End-labeling of DNA fragments}

DNA restriction fragments were end-labeled at the $3^{\prime}$ or $5^{\prime}$ ends using the large fragment of $E$. coli DNA polymerase I (Bethesda Research Labs, Inc.) or T4 polynucleotide kinase (Pharmacia, Inc.) after treatment with calf intestinal phosphatase (Boehringer-Mannheim, Inc.) according to standard protocols (Maniatis et al. 1982). After labeling, the fragments were phenol/chloroform/isoamyl alcohol- $(25: 24: 1)$ extracted, digested with a second restriction enzyme, and the appropriate fragment isolated by polyacrylamide electrophoresis. When necessary, fragments were purified on an Elutip-d column (Schleicher and Schuell, Inc.) following elution from the polyacrylamide gel.

\section{DNase I and DMS protection reactions}

End-labeled restriction fragments were incubated in a $50-\mu 1$ volume with the desired proteins under standard recombination conditions [ $(100 \mathrm{~mm} \mathrm{NaCl}, 20 \mathrm{~mm}$ Tris- $\mathrm{HCl}, \mathrm{pH} 7.6,5 \mathrm{~mm}$ $\mathrm{MgCl}_{2}$, and $1 \mathrm{mM}$ dithiothreitol (DTT)]. After $5 \mathrm{~min} 20-40 \mathrm{ng}$ of DNase I /gift of A. Newman/ was added and incubated for 30 sec and then quenched by the addition of $6 \mu$ lof $3.3 \%$ SDS, 66.7 mM trans-1,2-diaminocyclohexame- $N, N, N^{\prime}, N^{\prime}$-tetraacetic acid 
(CDTA) (Sigma), and $0.667 \mathrm{M}$ Tris-HCl $(\mathrm{pH} 9.5$ ) and freezing on dry ice. Samples were then digested with $20 \mu \mathrm{g}$ Proteinase $\mathrm{K}$ (Sigma) for $15 \mathrm{~min}$ at $37^{\circ} \mathrm{C}$, extracted with phenol/chloroform/ isoamyl alcohol- $(25: 24: 1)$ extracted, and ethanol-precipitated twice. Samples were taken up in recrystallized formamide and electrophoresed on $6 \%$ polyacrylamide geles containing 8 $M$ urea with a buffer gradient from $1 \times$ to $3 \times$ TBE $190 \mathrm{mM}$ Tris base, $90 \mathrm{~mm}$ boric acid, and $2.5 \mathrm{~mm}$ EDTA; Biggin et al. 1983). For DMS reactions, the DTT was omitted and the Tris- $\mathrm{HCl}$ was substituted with cacodylate- $\mathrm{NaOH}(\mathrm{pH}$ 7.8). The fragments were incubated for $2 \mathrm{~min}$ in $40 \mathrm{mM}$ DMS.

\section{DNA gel retardation assays}

EcoRI-HindIII restriction fragments from pMS551 (334 bp), pMS529 (249 bp), and pMS609 (326 bp) were end-labeled as described above. The concentration of the labeled DNA fragments was determined by comparison on an ethidium bromidestained agarose gel to DNA fragments of similar size and known concentration. The binding reactions were performed in $80 \mathrm{~mm} \mathrm{NaCl}, 25 \mathrm{~mm}$ Tris- $\mathrm{HCl}$ (pH 7.9), $1.2 \mathrm{~mm}$ EDTA, $2 \mathrm{~mm}$ DTT, $0.5 \mathrm{mg} / \mathrm{ml}$ bovine serum albumin (BSA), $0.2 \mathrm{mg} / \mathrm{ml}$ polycytidylic acid, and $15 \mu \mathrm{g} / \mathrm{ml}$ calf thymus DNA. Binding was initiated by addition of Fis (purified as described by Johnson et al. 1986) at the indicated concentrations (Fig. 4) to the reaction mixture containing the labeled fragment at a concentration of $2 \times 10^{-10} \mathrm{M}$ and incubated at room temperature for $20 \mathrm{~min}$. Both binding and electrophoresis of the Fis-enhancer complexes were performed as described by Bushman et al. (1984). Electrophoresis time and conditions were the same for each titration. The dried gels were exposed to Kodak XAR X-ray film at room temperature. The autoradiograms were scanned with a LKB 2202 Ultrascan laser densitometer and peak intensities were determined using the LKB2190-001 GelScan software package. Complexed and unbound DNA were also quantitated by cutting out the bands from the dried polyacrylamide gel and determining the Cerenkov counts for each band. Comparable results were obtained for each method and in separate experiments. The percent DNA bound at each enhancer domain by Fis was calculated by assuming for pMS609 and pMS529 that any retarded complex had the enhancer domain occupied and for pMS551 that complex 1 contained Fis bound only to the distal domain and all other retarded complexes had both domains occupied. This analysis ignores the small percentage of molcules with a secondary site occupied and an enhancer domain vacant.

Other gel binding assays described in the text were performed similarly using approximately 0.01 pmole of DNA fragment from the appropriate plasmids.

Titrations of Fis binding to enhancer DNA also were performed in the presence of a 100-fold molar excess of unlabeled fragment containing the enhancer; the same minimal amount of protein was required for the formation of complexes 1 and 2 as in the assays described above. This indicates that the affinity of Fis for the DNA was being measured in these assays rather than the amount of Fis present in the reaction mixture.

\section{Chemical interference assays}

HindIII-EcoRI (334 bp) and HindIII-EcoRV (276 bp) fragments from pMS551 were end-labeled at the $3^{\prime}$ or $5^{\prime}$ ends at the HindIII site, respectively, as described above. Approximately 0.1 pmole of labeled fragment was methylated using DMS (Aldrich) as described (Hendrikson and Schleif 1985). The binding reactions and electrophoresis to separate the protein-DNA complexes were performed as described above, incubating the methylated DNA fragments with approximately 50- to 100-fold molar excess of Fis protein (purified as described by Johnson et al. 1986); the control contained no added Fis. The complexed and unbound DNA were eluted from gel fragments, purified on Elutip-d columns, and ethanol-precipitated. Cleavage at the methylated purine residues was accomplished using the method described by Sakonju and Brown (1982). The cleaved DNA was resuspended in recrystallized formamide containing marker dyes and electrophoresed on $6 \%$ denaturing acrylamide gels (as described above) using samples prepared by the guanine sequencing reaction (Maxam and Gilbert 1977) as markers.

\section{Inversion assays}

DNA inversion assays were performed as described previously (Johnson et al. 1986).

\section{Acknowledgments}

We thank Carol Lee for technical assistance and Paul Kitts for useful discussions and critical reading of the manuscript. This work was supported in part by a grant from the National Science Foundation to M.I.S. M.F.B. was supported by a fellowship from the National Institutes of Health. A.C.G. is a fellow of the American Cancer Society, and R.C.J. was supported at Caltech by a Hoffman-LaRoche Fellowship from the Life Science Research Foundation and at UCLA by a grant from the Weingart Foundation.

\section{References}

Biggin, M.D., T.J. Gibson, and G.F. Hong. 1983. Buffer gradient gels and ${ }^{35} \mathrm{~S}$ label as an aid to rapid DNA sequence determination. Proc. Natl. Acad. Sci. 80: 3963-3965.

Boocock, M.R., J.L. Brown, and D.J. Sherratt. 1986. Structural and catalytic properties of specific complexes between Tn3 resolvase and the recombination site res. Biochem. Soc. Trans. 14: 214-216.

Boocock, M.R., J.L. Brown, and D.J. Sherratt. 1987. Topological specificity in $\mathrm{Tn} 3$ resolvase catalysis. In DNA replication and recombination, (ed. T.J. Kelley and R. McMacken), pp. 703-718. Alan R. Liss, New York.

Bruist, M.F., R.C. Johnson, M.B. Glaccum, and M.I. Simon. 1984. Characterization of Hin-dependent DNA inversion and binding in vitro. In Genome rearrangement (ed. M.I. Simon and I. Herskowitz), pp. 63-75. Alan R. Liss, New York.

Bushman, W., S. Yin, L.L. Thio, and A. Landy. 1984. Determinants of directionality in lambda site-specific recombination. Cell 39: 699-706.

Cozzarelli, N.R., M.A. Krasnow, S.P. Gerrard, and J.H. White. 1984. A topological treatment of recombination and topoisomerases. Cold Spring Harbor Symp. Quant. Biol. 49: $383-400$.

Craigie, R. and K. Mizuuchi. 1986. Role of DNA topology in $\mathrm{Mu}$ transposition: Mechanism of sensing the relative orientation of two DNA segments. Cell 45: 793-800.

Dynan, W.S. and R. Tjian. 1985. Control of eukaryotic messenger RNA synthesis by sequence-specific DNA-binding proteins. Nature 316: 774-778.

Fried, M. and D. Crothers. 1981. Equilibrium and kinetics of $1 a c$ repressor-operator interactions by polyacrylamide gel electrophoresis. Nucleic Acids Res. 9: 6505-6525.

Galas, D.J. and A. Schmitz. 1978. DNase footprinting: A simple method for the detection of protein-DNA binding specificity. Nucleic Acids Res. 5: 3157-3170.

Garner, M.M. and A. Revzin. 1981. A gel electrophoresis 
method for quantifying the binding of proteins to specific DNA regions. Applications to components of the Escherichia coli lactose operon regulatory system. Nucleic Acids Res. 9: 3047-3060.

Gellert, M. and H. Nash. 1987. Communication between segments of DNA during site-specific recombination. Nature 325: 401-404.

Gilbert, W., A. Maxam, and A. Mirzabekov. 1976. Contacts between the lac repressor and DNA revealed by methylation. In Control of ribosome synthesis (ed. N.O. Kjelgaard and O. Maaloe), pp. 139-148. Munksgaard, Copenhagen.

Grindley, N.D.F., M.R. Lauth, R.G. Wells, R.J. Wityk, J.J. Salvo, and R.R. Reed. 1982. Transposon-mediated site-specific recombination: Identification of three binding sites for resolvase at the res sites of gamma-delta and Tn3. Cell 30: 1927.

Hendrickson, W. and R. Schleif. 1985. A dimer of Ara C protein contacts three adjacent major groove regions of the ara I DNA site. Proc. Natl. Acad. Sci. 82: 3129-3133.

Hiestand-Nauer, R. and S. Iida. 1983. Sequence of the site-specific recombinase gene $\operatorname{cin}$ and of its substrates serving in the inversion of the $\mathrm{C}$ segment of bacteriophage P1. EMBO I. 2: $1733-1740$.

Huber, H.E., S. Iida, W. Arber, and T.A. Bickle. 1985. Site-specific DNA inversion is enhanced by a DNA sequence in cis. Proc. Natl. Acad. Sci. 82: 3776-3780.

Johnson, R.C. and M.I. Simon. 1985. Hin-mediated site-specific recombination requires two $26 \mathrm{bp}$ recombination sites and a 60 bp recombinational enhancer. Cell 41: 781-791.

Johnson, R.C., M.F. Bruist, M.B. Glaccum, and M.I. Simon. 1984. In vitro analysis of Hin-mediated site-specific recombination. Cold Spring Harbor Symp. Quant. Biol. 49: 751760.

Johnson, R.C., M.F. Bruist, and M.I. Simon. 1986. Host protein requirements for in vitro site-specific DNA inversion. Cell 46: $531-539$.

Johnson, R.C., A.C. Glasgow, and M.I. Simon. 1987. Mechanism of action of the Hin recombination enhancer. Nature (in press).

Kahmann, R., F. Rudt, C. Koch, and G. Mertens. 1985. G inversion in bacteriophage $\mathrm{Mu}$ DNA is stimulated by a site within the invertase gene and a host factor. Cell 41: 771780.

Kahmann, R., G. Mertens, A. Klippel, B. Brauer, F. Rudt, and C. Koch. 1987. The mechanism of $\mathrm{G}$ inversion. In DNA replication and recombination (ed. T.J. Kelley and R. McMaken), pp. 681-690. Alan R. Liss, New York.

Kanaar, R. and P. van de Putte. 1987. Topological aspects of site-specific DNA inversion. Bioessays (in press).

Koch, C. and R.K. Kahmann. 1986. Purification and properties of the Escherichia coli host factor required for inversion of the $\mathrm{G}$ segment in bacteriophage $\mathrm{Mu}$. I. Biol. Chem. 261: 15673-15678.

Maniatis, T., E.F. Fritsch, and J. Sambrook. 1982. Molecular cloning: A laboratory manual. Cold Spring Harbor Laboratory, Cold Spring Harbor, New York.

Maxam, A. and W. Gilbert. 1977. A new method for sequencing DNA. Proc. Natl. Acad. Sci. 74: 560-564.

Nash, H. and H.J. Pollock. 1983. Site-specific recombination of bacteriophage lambda: The change in topological linking number associated with exchange of DNA strands. $J$. Mol. Biol. 170: 19-38.

Pfeifer, K., T. Prezant, and L. Guarente. 1987. Yeast HAP1 activator binds to two upstream activation sites of different sequence. Cell 49: 19-27.

Plasterk, R.A., A. Brinkman, and P. van de Putte. 1983. DNA inversions in the chromosome of Escherichia coli and in bacteriophage $\mathrm{Mu}$ : Relationship to other site-specific recombination systems. Proc. Natl. Acad. Sci. 80: 5355-5358.

Ptashne, M. 1986. Gene regulation by proteins acting nearby and at a distance. Nature 322: 697-701.

Reed, R.R. 1981. Transposon-mediated site-specific recombination: A defined in vitro system. Cell 25: 713-719.

Sakonju, S. and D.D. Brown. 1982. Contact points between a positive transcription factor and the Xenopus 5S RNA gene. Cell 31: 395-405.

Sanger, F., S. Nicklen, and A.R. Coulson. 1977. DNA sequencing with chain-terminating inhibitors. Proc. Natl. Acad. Sci. 74: 5463-5467.

Siebenlist, U. and W. Gilbert. 1980. Contacts between Escherichia coli RNA polymerase and an early promoter of phage T7. Proc. Natl. Acad. Sci. 77: 122-126.

Simon, M., J. Zieg, M. Silverman, G. Mandel, and R. Doolittle. 1980. Phase variation: Evolution of a controlling element. Science 209: 1370-1374.

Suck, D. and C. Oefner. 1986. Structure of DNase I at $2.0 \mathrm{~A}$ resolution suggests a mechanism for binding to and cutting DNA. Nature 321: 620-625.

Thompson, J.F., L.M. de Vargas, C. Koch, R. Kahmann, and A. Landy. 1987. Cellular factors provide a mechanism for coupling recombination with growth phase: Characterization of a new component in the lambda site-specific recombination pathway. Cell (in press).

Zieg, J., M. Silverman, M. Hilmen, and M. Simon. 1977. Recombinational switch for gene expression. Science 196: $170-172$.

Zieg, J. and M. Simon. 1980. Analysis of the nucleotide sequence of an invertible controlling element. Proc. Natl. Acad. Sci. 77: 4196-4200. 


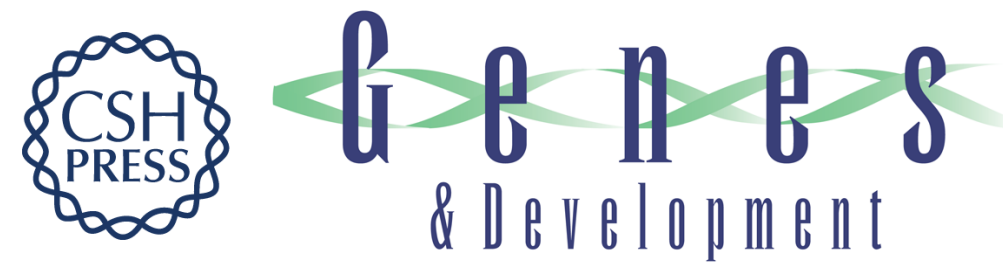

\section{Fis binding to the recombinational enhancer of the Hin DNA inversion system.}

M F Bruist, A C Glasgow, R C Johnson, et al.

Genes Dev. 1987, 1:

Access the most recent version at doi:10.1101/gad.1.8.762

$\begin{array}{ll}\text { References } & \text { This article cites } 32 \text { articles, } 14 \text { of which can be accessed free at: } \\ \text { http://genesdev.cshlp.org/content/1/8/762.full.html\#ref-list-1 }\end{array}$

License

Email Alerting Receive free email alerts when new articles cite this article - sign up in the box at the top Service right corner of the article or click here.

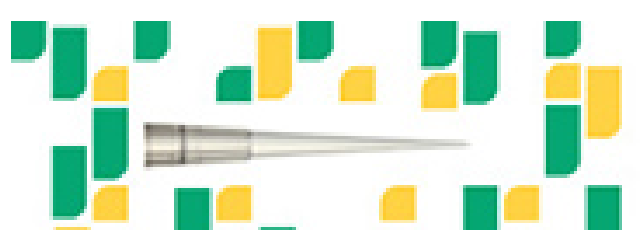

Focused on your science. 